\title{
Evaluation of Refractivity Profiles from CHAMP and SAC-C GPS Radio Occultation
}

Paul Poli ${ }^{1,4 *}$, Chi On Ao ${ }^{2}$, Joanna Joiner ${ }^{3}$, Manuel de la Torre Juárez ${ }^{2}$, Raymond Hoff ${ }^{1}$

${ }^{1}$ Joint Center for Earth Systems Technology, Baltimore, Md, USA

${ }^{2}$ California Institute of Technology, NASA Jet Propulsion Laboratory, Pasadena, Ca, USA

${ }^{3}$ Laboratory for Atmospheres, NASA Goddard Space Flight Center, Greenbelt, Md, USA

${ }^{4}$ also at Météo-France, Toulouse, France

The Global Positioning System enables positioning in 3 dimensions about the Earth. The signals sent by twenty-four satellites travel through our atmosphere down to the small receivers used by the civilian community and the military. These signals are radio waves; their travel path in the atmosphere is dependent upon varying meteorological conditions.

A small GPS receiver was launched in space with the GPS/Meteorology experiment (GPSMET) in 1995. For the first time, it became possible to perform a significant number of radio occultations around the Earth: from the standpoint of the receiver, the source - one of the 24 GPS satellites - would rise or set around the other side of the Earth. When the source would disappear, the receiver would progressively lose the signals. The delay induced by the atmosphere would then be inferred by measuring accurately the time delay between the emission and the reception of the signal.

In 2000, two second-generation GPS receivers were launched on-board the GeoForschungsZentrum's Challenging Mini-satellite Payload for Geophysical Research and Application (CHAMP, US-Germany) and the Comision Nacional de Actividades Especiales' Satelite de Aplicaciones Cientificas-C (SAC-C, US-Argentina). These second-generation "Blackjack" receivers have the ability to sense the lower troposphere closer to the surface than the proof-of-concept GPS/MET experiment. Since their launch, CHAMP and SAC-C have collected thousands of GPS radio occultations, representing a wealth of measurements available for data assimilation in Numerical Weather Prediction models.

In order to evaluate the data derived by the Jet Propulsion Laboratory from the raw radio occultation measurements of CHAMP and SAC-C, we use 6-hour forecasts produced by the Data Assimilation Office Finite Volume Data Assimilation System as an independent state of the atmosphere. We compare CHAMP and SAC-C refractivity retrievals with refractivity calculated from the forecast, i.e. global fields of temperature, water vapor content and humidity. We show statistics of the differences as well as histograms of the differences. Over one year of comparison, the statistics suggest a seasonal cycle in numerical forecast errors and/or processing errors (namely diffraction effects). This cycle appears stronger in the Northern hemisphere than in the Southern hemisphere.

*Corresponding author address: Paul Poli, Code 910.3, NASA/GSFC, Greenbelt, MD 20771. e-mail: ppoli@dao.gsfc.nasa.gov 


\section{Evaluation of refractivity profiles from CHAMP and SAC-C GPS radio occultation}

Paul Poli ${ }^{1,2,4}$, Chi On $\mathrm{Ao}^{3}$, Joanna Joiner ${ }^{1}$, Manuel de la Torre Juárez ${ }^{3}$, and Raymond Hoff ${ }^{2}$

1 Data Assimilation Office, NASA Goddard Space Flight Center, Greenbelt MD 20771 ppoli@dao.gsfe. nasa.gov

2 Joint Center for Earth Systems Technology, University of Maryland Baltimore County, Baltimore MD 21250

${ }^{3}$ California Institute of Technology, NASA Jet Propulsion Laboratory, Pasadena, CA 91109

4 also at Météo France Centre National de Recherches Météorologiques, Toulouse, France

Summary. The GeoForschungsZentrum's Challenging Minisatellite Payload for Geophysical Research and Application (CHAMP, Germany-US) and the Comision Nacional de Actividades Especiales' Satelite de Aplicaciones Cientificas-C (SAC-C, Argentina-US) missions are the first missions to carry a second-generation Blackjack Global Positioning System (GPS) receiver. One of the new features of this receiver is its ability to sense the lower troposphere closer to the surface than the proof-of-concept GPS Meteorology experiment (GPS/MET). Since their launch, CHAMP and SAC-C have collected thousands of GPS radio occultations, representing a wealth of measurements available for data assimilation and Numerical Weather Prediction (NWP). In order to evaluate the refractivity data derived by the Jet Propulsion Laboratory (JPL) from raw radio occultation measurements, we use Data Assimilation Office (DAO) 6-hour forecasts as an independent state of the atmosphere. We compare CHAMP and SAC-C refractivity (processed by JPL) with refractivity calculated from the DAO global fields of temperature, water vapor content and humidity. We show statistics of the differences as well as histograms of the differences.

Key words: Radio-occultation - Refractivity - Skewness - Seasonal cycle

\section{Introduction}

The CHAMP and SAC-C missions have each collected more than 30,000 GPS radio occultations since May 2001 (July 2001, respectively). Theoretical studies [HE00] as well as studies based on GPS/MET data [PJK02] have suggested that GPS radio occultation data could have a potential impact on data assimilation applied to Numerical Weather Prediction (NWP) and/or climate 
studies. With the wealth of GPS radio occultation data now available, the NWP community is now given an amount of observations which compete in number with other observing systems (e.g. radiosondes) with, for example, the advantage that radio occultation observations collected by a high-inclination Low-Earth Orbiter (LEO) are distributed more evenly on our planet.

In the present paper, we compare DAO 6-hour forecasts with CHAMP and SAC-C refractivity, for a total of $\sim 54,000$ occultations. We also investigate the distribution of the differences in terms of histograms to potentially verify the usual assumptions used in variational retrievals and data assimilation (Gaussian unbiased errors).

\section{Methodology}

In order to evaluate the refractivity derived from CHAMP and SAC-C, we investigate time, latitude and altitude dependence of the differences between DAO 6-hour forecasts and refractivity derived from radio occultation. We focus here on 6-hour forecasts because our interest is future assimilation of refractivity into latest available 6-hour forecasts. All the refractivity differences are DAO forecasts minus (derived from radio occultation) (or "observed"), expressed in percent of observed refractivity, in order to account for the fact that refractivity decays exponentially with altitude and spans about two orders of magnitude between the surface and $30 \mathrm{~km}$ altitude.

\subsection{Refractivity Derived from DAO Forecasts}

We ran the DAO Finite Volume Data Assimilation System between April 2001 and April 2002 with a horizontal resolution of $2 \times 2.5$ degrees (lat/lon) and 55 levels between the surface and $0.01 \mathrm{hPa}$. Conventional observations (including radiosondes) as well as (A)TOVS brightness temperature and SSM/I precipitable water were assimilated. No GPS radio occultation observations were assimilated in this run. We derived local refractivity at the tangent point latitude/longitude/altitude reported in each occultation by applying the formula derived by Smith and Weintraub [SW53]. We did not perform any horizontal averaging to account for the effects of horizontal refractivity gradients.

\subsection{Refractivity Derived from CHAMP and SAC-C}

We used the refractivity data derived by JPL, available through an anonymous FTP website. Details of the geometrical optics processing employed to process the raw radio occultation measurements can be found in the literature [HA02]. All occultations presenting more than $20 \%$ difference in refractivity with respect to NCEP analyses were rejected by the data producers. We did not apply any further quality control to the dataset. Table 1 shows the number of occultations used in our study. 
Table 1. Number of radio occultations.

\begin{tabular}{lccccccccccccc}
\hline \multicolumn{1}{c}{ May01 Jun01 Jul01 Aug01 Sep01 Oct01 Nov01 } & Dec01 Jan02 Feb02 Mar02 Apr02 \\
\hline CHAMP & 2295 & 3284 & 2352 & 1157 & 1715 & 2717 & 2666 & 1995 & 3245 & 2698 & 3785 & 2175 \\
SAC-C & - & - & 824 & 3249 & 3994 & 2835 & 2666 & 2339 & 3183 & - & 3467 & 1769 \\
\hline
\end{tabular}

\subsection{Origins of the Refractivity Differences}

The refractivity differences we are looking at in the present paper arise from three error types: representativeness, observation, and forecast errors. The first errors include limitations in our observation operator (assumes local refractivity and does not account for horizontal gradients). The term 'observation errors' covers "GPS radio occultation"-related issues, many of which are discussed by other authors in the present volume (e.g. limitations of the geometrical optics processing, hardware and software tracking of the occultations etc.). Eventually, forecast errors represent a motivation for assimilating GPS radio occultation data in NWP.

\section{Mean and Standard Deviation of the Differences: Large-Scale Space and Time Variations}

It is primordial for the purposes of data assimilation to use adequate errors in order to ensure adequate weighting of the observations versus other sources of information (i.e. numerical forecast, other observations). These errors are usually modeled as Gaussian errors in data assimilation systems, thus requiring a priori knowledge of the mean departure (bias) as well as the standard deviation.

We investigate first the spatial variations of the differences at a given time (January 2002, entire month). Figure 1a shows the bias for the CHAMP minus DAO refractivity. This bias has already been reported by several authors [e.g. [RAE97]]. Its origins remain unclear. The magnitude of the bias decreases with altitude and is more important in the lower atmosphere, where water vapor is more abundant. We checked that the regions of higher bias move with latitude and seasons. Figure $1 \mathrm{~b}$ is the same plot but for SAC-C refractivity. It is reassuring to notice very similar patterns, except for a small $-0.5 \%$ bias in the Tropics between 7 and $20 \mathrm{~km}$ altitude.

Figure 1c shows zonal averages for the standard deviation of the differences. Those are larger in the lower layers where horizontal gradients play a larger role. The numbers agree with the SAC-C results shown in Figure 1d.

Figure 2a shows the variations in the standard deviations throughout one year (from May 2001 until April 2002) for CHAMP, at 5.5km altitude. For a set latitude in the Northern hemisphere $\left(45^{\circ} \mathrm{N}\right.$ for example), a seasonal cycle can be seen: larger standard deviations occur in July. This cycle appears 

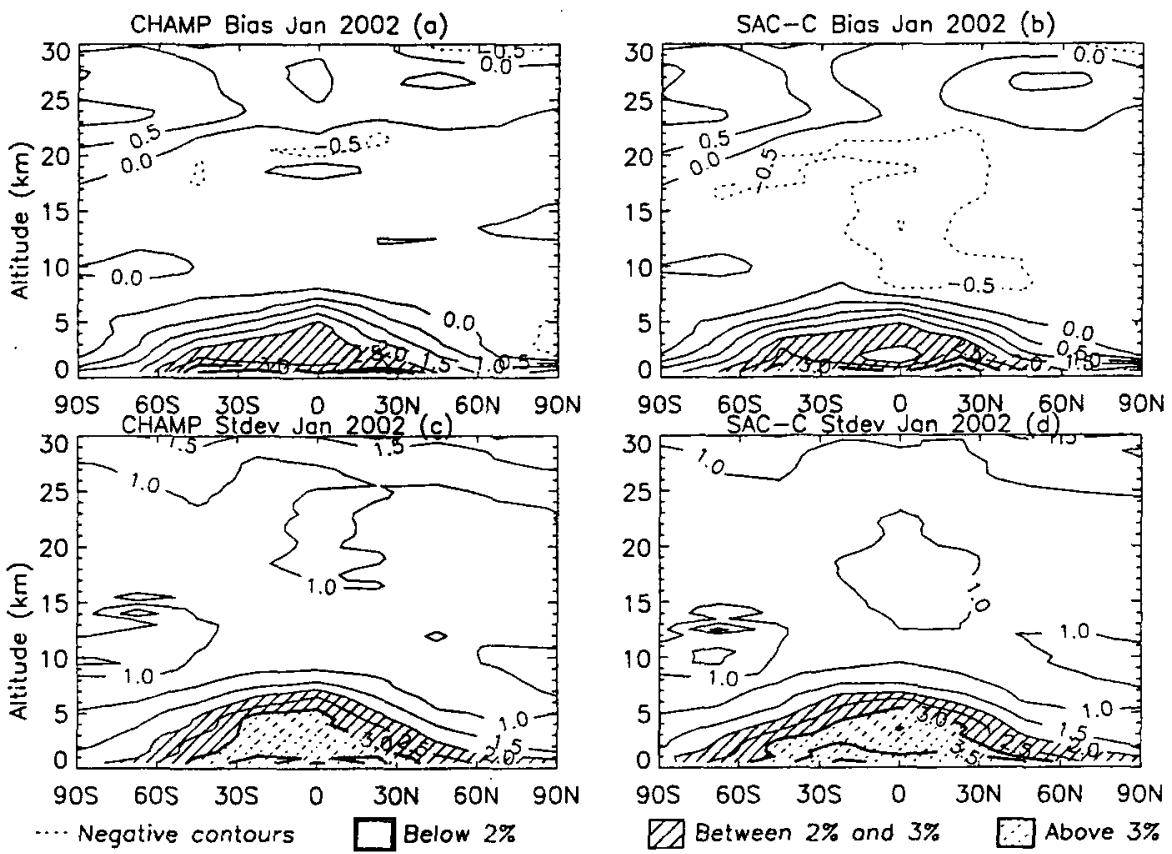

Fig. 1. Zonal averages of bias and standard deviation of the differences between CHAMP (SAC-C) and DAO 6-hour forecasts refractivity. Contour every $0.5 \%$. See text for details.

smaller in the Southern hemisphere but the idea is similar. Larger standard deviations are observed in the summer hemisphere. Figure $2 \mathrm{~b}$ shows similar results for SAC-C (note: no data for May and June 2001, and missing data for February 2002). Figure 2c is for a lower altitude $(3.5 \mathrm{~km})$, where more humidity is present. Humidity may play a role in the refractivity differences through the three sources of errors. Consequently, it is not possible to sort out the origin of the seasonal cycle shown here. The seasonal cycle appears definitely stronger in the Northern hemisphere than in the South. These conclusions are reinforced by looking at Figure 2d (for SAC-C), as well as Figures $2 \mathrm{e}$ and $2 \mathrm{f}$ (2.5 $\mathrm{km}$ altitude).

\section{Histograms of the Refractivity Differences}

As stated earlier, assimilation methods usually assume Gaussian errors. We attempt to verify this assumption by inspection of histograms of the refractivity differences.

In order to account for the fact that polar regions cover a smaller area than tropical regions, we included an area-weighting factor in the calculations. It is to be noted that this did not alter fundamentally the results. Figure 3a shows 

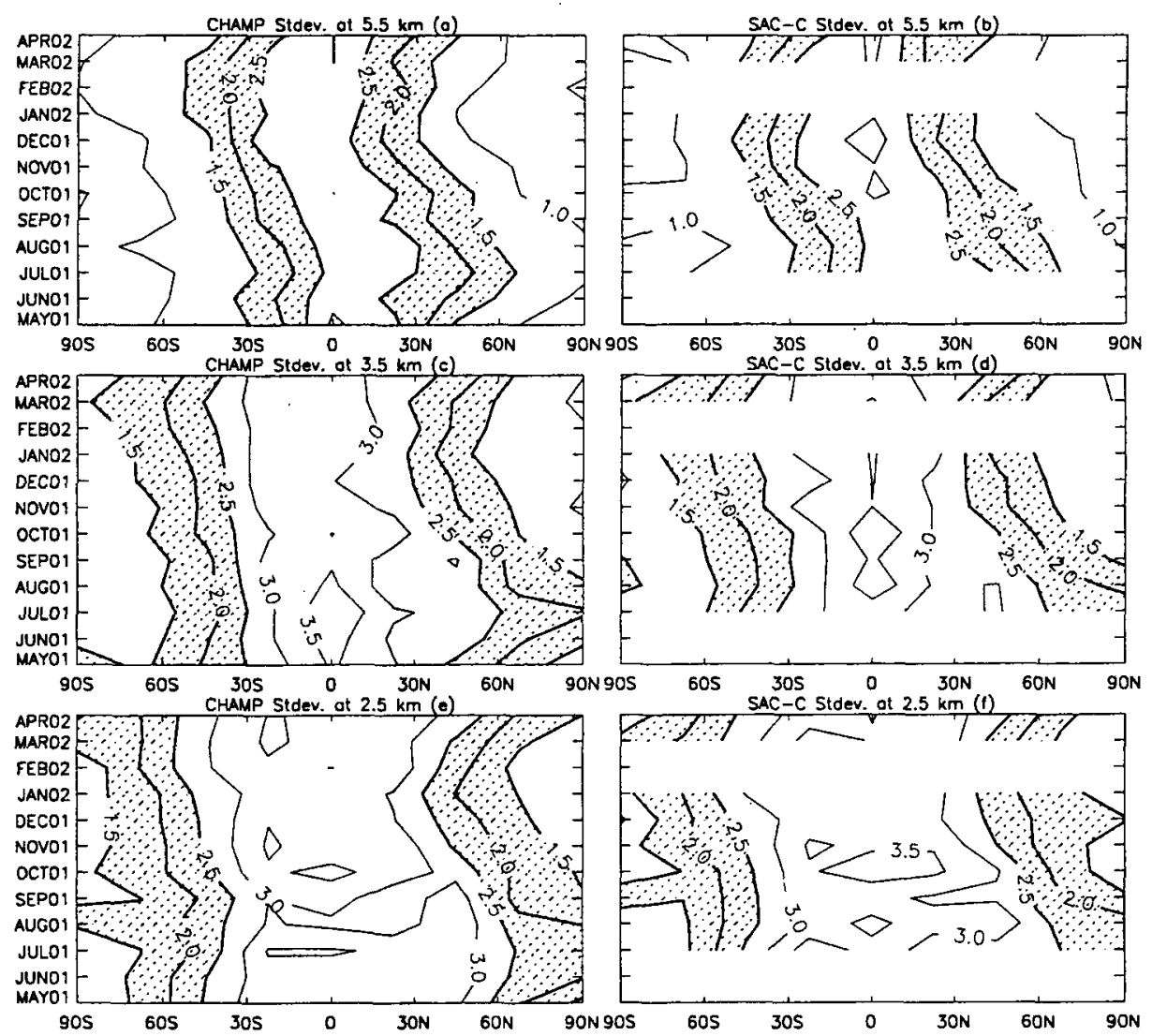

Fig. 2. Time evolution of the quantities shown in Figure 1 for several altitudes $(2.5 \mathrm{~km}, 3.5 \mathrm{~km}$, and $5.5 \mathrm{~km}$, standard deviations only). Shaded area: standard deviation between $1.5 \%$ and $2.5 \%$.

the repartition of refractivity differences (DAO minus CHAMP) for all occultations between May 2001 and April 2002, at $2 \mathrm{~km}$ altitude. The histogram in Figure 3a exhibits a skewed shape. The mode of the distribution is at zero, which indicates that the maximum likelihood is encountered for differences of zero. However, events with a positive differences are more common than events with a negative difference, thus leading to a skewness, and then a bias $(1.3 \%)$ in the distribution. The Gaussian fit, also plotted in Figure 3a, shows the positive bias which results from the skews distribution.

For an altitude of $12 \mathrm{~km}$, Figure $3 \mathrm{~b}$ shows that the skewness has disappeared. The bias is relatively small (less than $0.1 \%$ ). However, a departure from the Gaussian shape is apparent for zero differences: such events are more frequent in reality than modeled by a Gaussian curve.

We investigated the latitudinal dependence of the skewness illustrated in Figure 3a. No clear conclusion could be drawn. It remains that the skewness 
is limited to lower portions of the atmosphere. Although-not shown here, we obtained very similar results by looking at histograms of DAO minus SAC-C refractivity.

(a) CHAMP 2000m (24235)

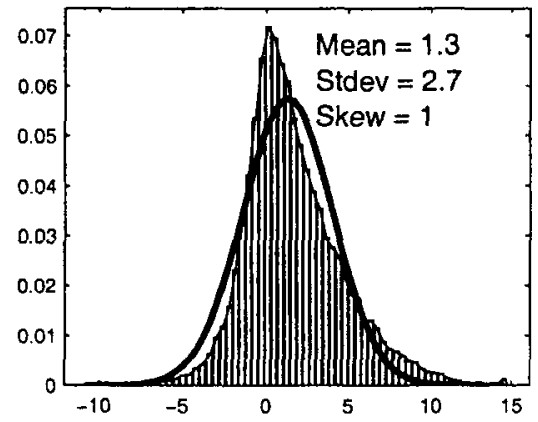

(b) CHAMP 12000m (29577)

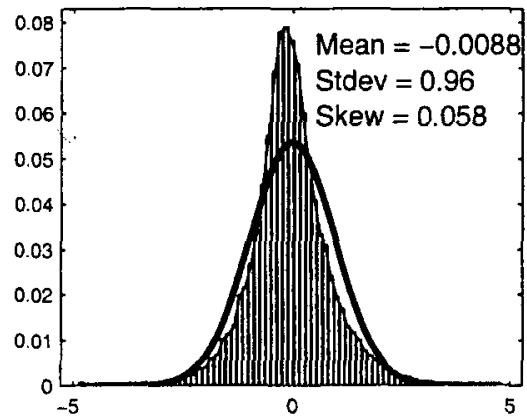

Fig. 3. Histograms of the refractivity differences for CHAMP, at two different altitudes, (May 2001-April 2002, all latitudes). Thick line is Gaussian fit. Number of comparisons in parentheses.

\section{Conclusions and Future Directions}

This study compares about 30,000 CHAMP and 24,000 SAC-C refractivity profiles with DAO 6-hour forecasts. The results obtained in this study will be used to refine error characteristics in order to perform one-dimensional variational analyses of the refractivity profiles derived by radio occultation. It seems that the refractivity differences do not vary with much intensity over time, although a seasonal cycle in the differences is observed for mid-latitudes in both hemispheres (apparently stronger in the Northern hemisphere than in the South). The refractivity differences show fairly constant means and standard deviations between 12 and $28 \mathrm{~km}$ altitude.

The histograms of refractivity differences present skewed distributions. It is not clear for now if this is due to the processing technique, insufficient modeling in our observation operator, or if it is a feature of the DAO model errors. In any case, this skewness seems to be related with the "refractivity bias". It deserves more attention.

\section{References}

[HA02] Hajj, G.A., Kursinski, E.R., Romans, L.J. et al.: A technical description of atmospheric sounding by GPS occultation. J. Atmos. Sol-Terr. Phys. 64, 451-469 (2002) 
[HE00] Healy, S.B., Eyre, J.R.: Retrieving temperature, water vapour and surface pressure information from refractive index profiles derived by radio occultation: a simulation study. Quart. J. Roy. Met. Soc., 126, 1661-1683, Part A (2000)

[PJK02] Poli, P., Joiner, J., Kursinski, E.R.: 1DVAR analysis of temperature and humidity using GPS radio occultation refractivity data. J. Geophys. Res., Accepted (2002)

[RAE97] Rocken, C., Anthes, R.A., Exner, M., Hunt, D., Sokolovskiy, S., Ware, R.H., Gorbunov, M.E., Schreiner, W., Feng, D., Herman, B., Kuo, Y.H., Zou, X.: Analysis and validation of GPS/MET data in the neutral atmosphere. J. Geophys. Res., 102, 29,849-29,866 (1997)

[SW53] Smith, E.K., Weintraub, S.: The constants in the equation for atmospheric index at radio frequencies. Proc. IRE, 41, 1035-1037 (1953) 